\title{
Computational Thermal Model of Unidirectional Composites with Random Fiber Array
}

\author{
Yuxuan Zhang ${ }^{1}$, Hui Wang ${ }^{1, a}$ and Wanqing Lin ${ }^{1}$ \\ ${ }^{1}$ Department of Engineering Mechanics, Henan University of Technology, Zhengzhou 450001, China
}

\begin{abstract}
The purpose of this work was to study the influence of microstructure on effective transverse thermal behavior of unidirectional fiber reinforced composites. Three types of microstructures are taken into account, including square periodic, hexagonal periodic and random arrangements of circular fibers. Unlike classical results at low fiber volume fractions and low thermal conductivity contrast between fibers and matrices, results provided by finite elements simulations for copper matrix composite reinforced with Carbon T-300 fibers have shown that random microstructures strongly affect the effective thermal properties of unidirectional composites for both high volume fractions and thermal conductivity contrast and can give closer predictions to the experimental results than the regular microstructures and the theoretical model.
\end{abstract}

\section{Introduction}

Unidirectional fiber reinforced composites [1] used for most engineering applications can be regarded as homogeneous materials with transversely isotropic/anisotropic properties from a macroscopic view. Moreover, it has been revealed that the macro-level behavior of unidirectional composite is usually controlled by its microstructure including constituent properties, fiber volume fraction, fiber shape as well as fiber arrangement. Usually, for the purpose of simplicity, the fiber arrangement in unidirectional composite follows an assumption that the fibers are dispersed in the matrix with regular pattern, i.e. the square pattern or the hexagonal pattern, so that a simple micromechanical unit cell model can be taken out from the composite for further homogenization analysis [2-4]. However, such assumption is different to the real distribution of fibers in the matrix material. It has been observed that the real microstructures are often random [5]. Therefore, how to assess the influence of randomly dispersed fibers to the effective thermal properties of composites have to be paid more attention [6].

Actually, at low fiber volume fraction and weak material property contrast between phases, the random composites can give rather similar predictions of overall properties of composite as the regular composites $[7,8]$. But for high fiber volume fraction, i.e. $40 \%$ and more, and high thermal property contrast between matrix and fiber, there is still a doubt that microstructure influences effective thermal conductivity of composites.

Present work is a comparison between transverse effective thermal conductivity of unidirectional fiber reinforced composite respectively built with square, hexagonal and random distribution of nonoverlapping circular fibers, for a large range of fiber volume fraction and thermal property contrast.

\footnotetext{
${ }^{a}$ Corresponding author : huiwang@haut.edu.cn
} 
The homogenized effective thermal conductivities of these composites are evaluated by finite element simulations followed by an area averaging of local thermal properties.

\section{Generation of random pattern of fibers}

For an unit cell of unidirectional composite, the relation of the fiber volume fraction, the number of fibers, the fiber size and the unit cell size can be written as

$$
v_{f}=\frac{n_{f} \times \pi r_{f}^{2}}{a \times a}
$$

from which one can have

$$
a=r_{f} \sqrt{\frac{n_{f} \times \pi}{v_{f}}}
$$

where $v_{f}, r_{f}$ and $n_{f}$ are the fiber volume fraction, fiber radius and number of fibers in the unit cell. $a$ is the side length of square unit cell.

Generally speaking, the fiber volume fraction and fiber radius are known parameters in practice for a known unidirectional composite, thus just $n_{f}$ and $a$ can be adjustable for establishing micromechanical unit cell model. From this point of view, the side length $a$ of the square unit cell increases in terms of the number of fibers $n_{f}$ in the unit cell, that is, how large the unit cell is.

To automatically generate random distribution of fibers in the unit cell to characterize their real case in composite as much as possible and simultaneously, avoid the fiber intersecting with the boundary of cell, the following algorithm is designed to produce the position of circular center by using random number

$$
\left[x_{c}, y_{c}\right]=\operatorname{rand}(1,2) \times\left(a-2 r_{f}\right)+r_{f}
$$

where rand(1,2) is an inbuilt function in MATLAB to produce random point in the $[0,1] \times[0,1]$ region, and $\left[x_{c}, y_{c}\right]$ is the location of circular fiber center. Moreover, the fibers can't overlap actually, thus the distance between any two fibers should exceed two times of fiber radius.

Repeating such procedure until the prescribed number of fibers is generated in the unit cell. To demonstrate this algorithm, let's consider an example. If the fiber radius is taken as $3.5 \mu \mathrm{m}$, and the fiber volume fraction is set to be $50 \%$, the random arrays with 100 and 400 fibers can be automatically produced by the present algorithm, as illustrate in Figure 1. It is found that the size of unit cell becomes bigger with the increase of the number of fibers, as expected from Eq. (2). Moreover, one can see that the 400 fibers are more densely packed than the 100 fibers.
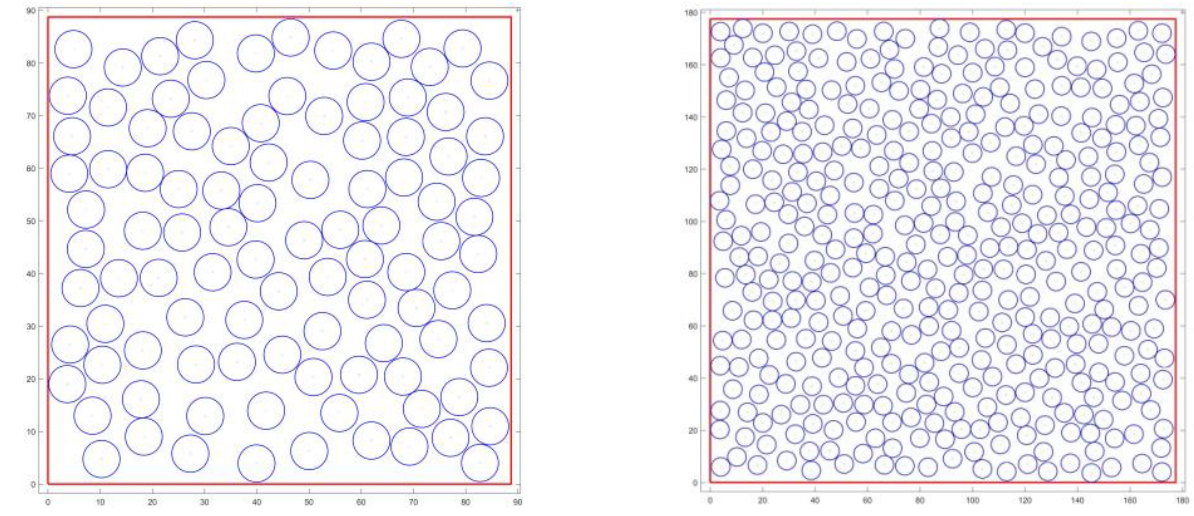

Figure 1. Two random arrays of fibers in the unit cell with 100 fibers (left) and 400 fibers (right) 


\section{Computational models}

The composite under consideration is all reinforced with unidirectional circualr fibers of same diameters. Apart from the fiber volume fraction and the thermal property contrast between material phases, the only difference between samples studied is the fiber transverse distribution which could be distributed randomly or regularly in square or hexagonal pattern.

Determination of homogenized effective transverse elastic properties of these composites requires samples large enough to ensure statistical homogeneity. Due to its periodicity, the unit cell for the square patrtern and the hexagonal pattern of fibers is respectively reduced to a square cell surrounding a single fiber and a square cell embedding two fibers, as shown in Figure 2. While, for random case, the unit cell have to be chosen to be big enough, in other words, contain a sufficient number of fibers, so that the overall thermal conductivities are approximately independent of the applied boundary conditions [9].

In the following computational analysis, the number of fibers are 100 and 400, respectively for random composites, as illustrated in Figure 1. Corrspondingly, the regualr composite is assumed to have same fiber volume fraction for the purpose of comparison. The fiber is taken as T-300 Carbon fiber, which has diameter $7 \mu \mathrm{m}$ and transverse thermal conductivity $0.8 \mathrm{~W} / \mathrm{mK}$. The matrix material is copper, which has thermal conductivity $380 \mathrm{~W} / \mathrm{mK}[10]$.

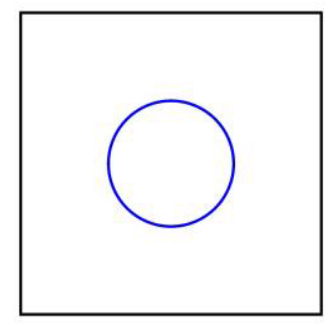

(a) Square array

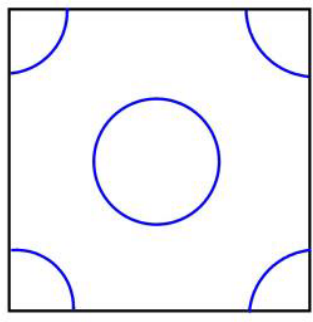

(b) Hexagonal array

Figure 2. Regular arrays of fibers in the matrix

\subsection{Effective thermal conductivity}

For the unidirectional composite reinforced with long fibers, the transverse heat transfer can be regarded as the two-dimensional heat transfer in the $x y$ plane. For such case, the temperature $(T)$ at any local point in the unidirectional composite unit cell shown in Figure 3 must satisfy the governing equation in both the matrix and the fiber

$$
\frac{\partial^{2} T}{\partial x^{2}}+\frac{\partial^{2} T}{\partial y^{2}}=0
$$

The boundary conditions corresponding to Eq. (4) may be written as

$$
\begin{aligned}
& k \frac{\partial T}{\partial y}=0 \text { at } y=0 \text { and } y=a \\
& T=T_{1} \text { at } x=0 \\
& T=T_{2} \text { at } x=a
\end{aligned}
$$

where $k$ is the thermal conductivity of either the fiber or the matrix.

In addition to the boundary conditions given in Eq. (5), the conditions existing at the interface between the fiber and the matrix should be specified. For zero thermal resistance at the interface

$$
\begin{aligned}
T_{f} & =T_{m} \\
\left.k_{f} \frac{\partial T}{\partial n}\right|_{f} & =\left.k_{m} \frac{\partial T}{\partial n}\right|_{m}
\end{aligned}
$$


where $n$ symbolizes the direction normal to the interface.

One can now solve the above problem consisting of Eqs. (4)-(6). Having obtained a solution to this problem, one would first calculate the local heat transfer rate at points along the surface $x=a$ and then an average heat transfer rate $\tilde{q}$ can be computed. Using this average value, a composite thermal conductivity will be now defined as [11]

$$
k_{x}=\frac{\tilde{q} a}{\Delta T}
$$

Here $\Delta T$ is the temperature difference between the isothermal surfaces $x=0$ and $x=a$, as indicated in Figure 3.

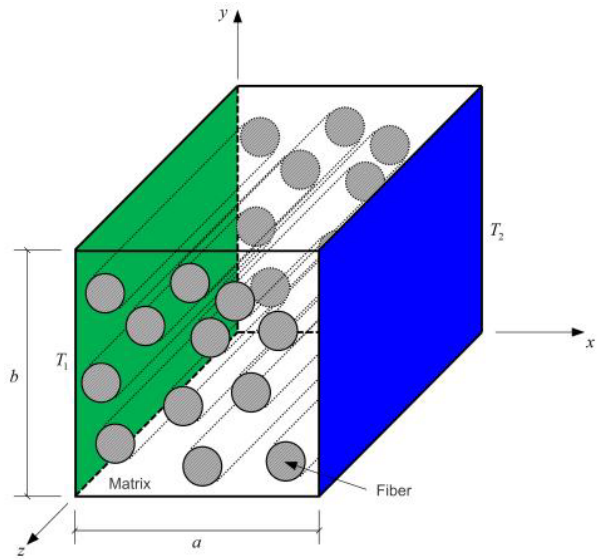

Figure 3. Transverse heat transfer in the unit cell

\subsection{Meshing convergence}

In the study, all composite samples are meshed by triangular-shaped linear finite elements. In order to ensure accurate results, even in high thermal gradient situations, the finite element mesh must be fine enough to avoid dependence of results with mesh scales. Different meshes are tested with regard to mesh densities. Figure 4 presents results of convergence for random unit cells with fiber volume fraction $50 \%$. In both configurations the final mesh was fined enough to represent accurately the geometry of inclusions. It is observed that the required convergent number of elements dramatically increase for the random sample with 400 fibers, compared to the random sample with 100 fibers.
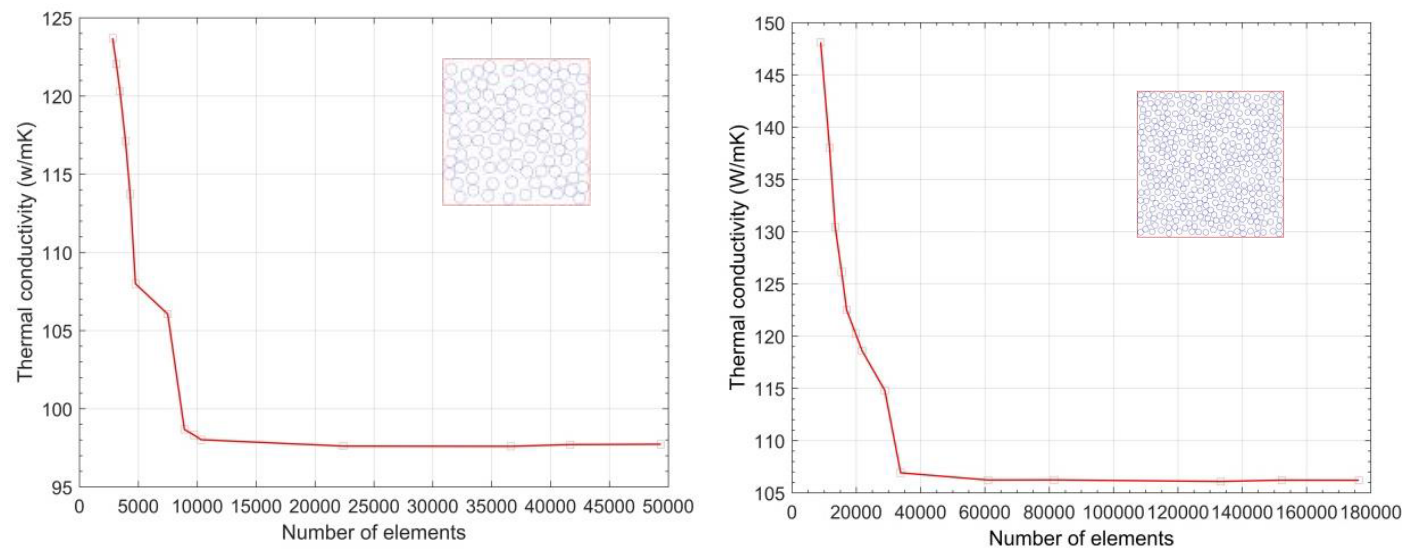

Figure 4. Effect of mesh density on the effective transverse thermal conductivity for the random microstructures with 100 fibers (left) and 400 fibers (right) 


\subsection{Results}

In this subsection, the influences of fiber volume fraction and the thermal property contrast are investigated for both the regualr and random samples. Here, the regular arrays of fibers including the square array and the hexagonal array (see Figure 2) are compared. Besides, the theoretical prediction from the Gurtman model [10] which is based on a periodic and regular composite unit cell theory is also provided in Eq. (8) for performing comparison.

$$
k_{x}=v_{f} k_{f}\left(1+v_{m} A\right)+v_{m} k_{m}\left(1-v_{f} A\right)
$$

where

$$
A=\frac{k_{m}-k_{f}}{\left(k_{m}+k_{f}\right)\left(1+v_{f}\right)}
$$

where $k_{m}$ and $k_{f}$ are thermal conductivity of fiber and matrix, respectively, and $v_{m}=1-v_{f}$.

\subsubsection{Effect of fiber volume fraction}

For various fiber volume fractions, i.e. changing from $5 \%$ to $50 \%$, which cover the low value to the high value, the effective thermal conductivity of composite is evaluated. The number of fibers in the unit cell is chosen as 100 and 400 for random samples, 2 for regular hexagonal sample and 1 for regular square sample. Results in Figure 5 indicate that the random distribution of fibers gives different results to the theoretical prediction, while both the square and hexagonal arrays seem to predict well the transverse thermal conductivity of unidirectional metal matrix composites. From Figure 5, it is also resulting that the transverse thermal conductivity obtained from random samples is much lower than those from theoretical values and regular samples, and the discrepancy becomes more significant with the increase of fiber volume fractions. But, it is found that the results from random models are closer to the experimental results then the theoretical and regular predictions. Moreover, one can see that both the two random samples give slightly different predictions at high fiber volume fractions. Besides, it is observed from Figure 5 that the transverse thermal conductivity of composites nonlinearly decreases with the increase of fiber volume fraction. This can be attributed to the poor conductivity of T-300 fibers.
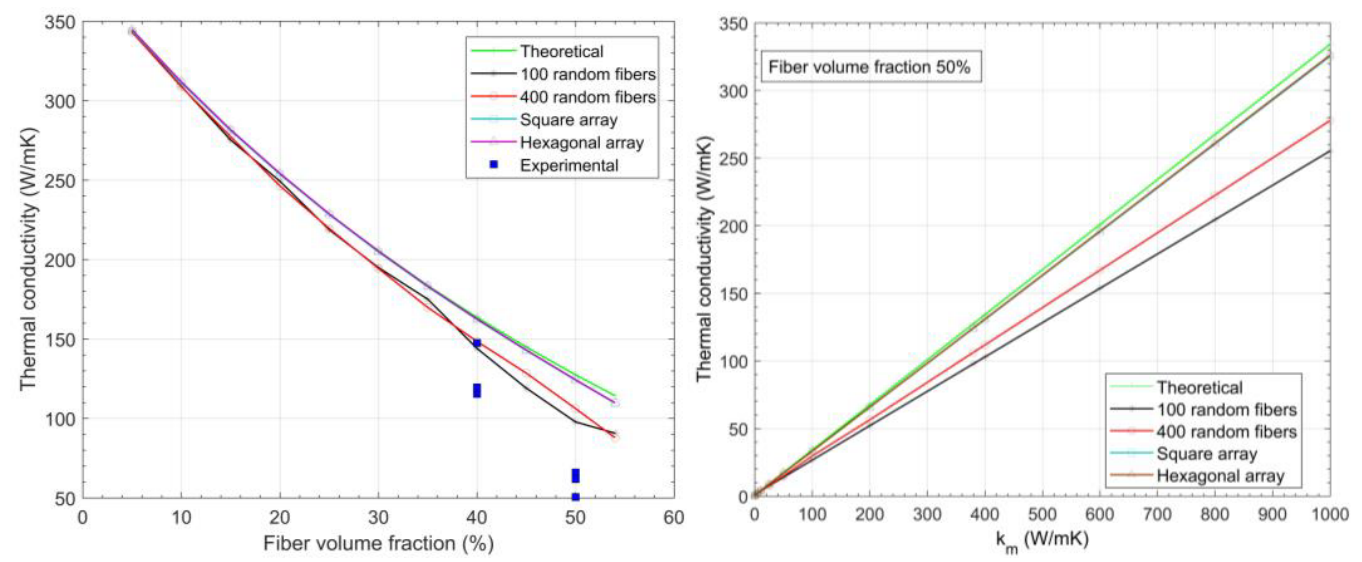

Figure 5. Effects of fiber volume fraction (left) and property contrast (right)

\subsubsection{Effect of property contrast}

To investigate the effect of property contrast of fiber and matrix materials, it is assumed that the thermal conductivity of fiber keeps $0.8 \mathrm{~W} / \mathrm{mK}$ unchanged while the thermal conductivity of matrix 
changes from 1000 to $0.1 \mathrm{~W} / \mathrm{mK}$. The fiber volume fraction is $50 \%$. One can see from Figure 5 that all samples including the regular samples and the random samples give lower prediction than the theoretical equation(8), and the regular samples can give the closer results to the theoretical predictions, compared to the random samples, which have inhomogeneous fiber distributions. But the discrepancy of different predictions becomes larger as the $k_{m}$ becomes bigger. In addition, in Figure 5, it is presented that the transverse thermal conductivity of unidirectional composites linearly varies in terms of the $k_{m}$.

\section{Conclusions}

The study investigates the influence of fiber distribution in the matrix by comparing random fiber arrays and regular fiber arrays. The random samples generated by a simple algorithm are used to approximate the real status of fibers in unidirectional composite. The results shows that the transverse thermal conductivity of unidirectional copper-matrix composites with Carbon fibers nonlinearly decreases from about 343 to about $87 \mathrm{~W} / \mathrm{mK}$ when the fiber volume fraction increases from $5 \%$ to $54 \%$, due to the poor thermal conductivity of T-300 fibers, whilst it linearly increases in terms of the matrix's thermal conductivity. More importantly, the obvious discrepancy can be observed between the random samples, the regular samples and the theoretical prediction. The random samples can give closer results of transverse thermal conductivity of unidirectional composites to the experimental results. This can be attributed to the inhomogeneous distributions of fibers. Denser packing of fibers unavoidably causes more complex behaviour of thermal transfer in composites, compared to the regular distributions.

\section{Acknowledgements}

The research work is partially supported by the Natural Science Foundation of China (grant no. 11472099) and the Program for Innovative Research Team of Science \& Technology of Henan Province of China (Grant no. 19IRTSTHN020).

\section{References}

1. P.K. Mallick, Fiber-Reinforced Composites: Materials, Manufacturing, and Design (CRC Press, 2007)

2. H. Wang, Q.H. Qin, Appl. Math. Comput. 268, 311-321 (2015)

3. H. Wang, Q.H. Qin, Y. Xiao, Int. J. Heat Mass Tran. 92, 228-235 (2016)

4. H. Wang, X.J. Zhao, J.S. Wang, Compos. Sci. Technol. 118, 117-126 (2015)

5. A.A. Gusev, P.J. Hine, I.M. Ward, Compos. Sci. Technol. 60 (4), 535-541 (2000)

6. B.Q. Kang, P. Yan, C.P. Jiang, Acta Metall. Sin-Engl. 29 (5), 140-145 (2012)

7. H. Wang, Q.H. Qin, Eng. Computation. 28 (8), 1079-1097 (2011)

8. D. Trias, J. Costa, J.A. Mayugo, J.E. Hurtado, Comp. Mater. Sci. 38 (2), 316-324 (2006)

9. T. Kanit, S. Forest, I. Galliet, V. Mounoury, D. Jeulin, Int. J. Solids Struct. 40 (13), 3647-3679 (2003)

10. J. Koráb, P. Štefánik, Š. Kavecký, P. Šebo, G. Korb, Compos. Part A. 33 (4), 577-581 (2002)

11. G.S. Springer, S.W. Tsai, J. Compos. Mater. 1, 166-173 (1967) 